\title{
Patrones de Discurso Observados en el Aula de Matemática de Segundo Ciclo Básico en Chile
}

\section{Discourse Patterns Observed in Middle-School Level Mathematics Classes in Chile}

\author{
Darinka Radovic y David Preiss \\ Pontificia Universidad Católica de Chile
}

\begin{abstract}
Las secuencias triádicas (pregunta-respuesta-seguimiento) son una forma frecuente de interacción en las salas de clases de diversas partes del mundo. A partir de la codificación del discurso hablado, el presente estudio describe estas secuencias en 89 clases de matemáticas de segundo ciclo básico de escuelas públicas filmadas en Chile, elegidas al azar de 684 clases filmadas para una evaluación nacional. En base al análisis descriptivo de las frecuencias con la que los profesores utilizan distintos tipos de preguntas y seguimientos y a la asociación de estas con actividades de la clase y desempeño del profesor, se identificó un patrón de interacción relativamente compartido, caracterizado por preguntas cerradas, escasa participación de los estudiantes y seguimientos de bajo potencial metacognitivo. Se evidenció que, al ejecutar actividades de práctica de contenidos matemáticos, los profesores produjeron más preguntas de aplicación y que los profesores mejor evaluados produjeron mayor cantidad de preguntas abiertas y sobre experiencias personales. Los resultados sugieren que una potencial área de capacitación de profesores es el aumento del repertorio del discurso pedagógico.
\end{abstract}

Palabras clave: discurso, enseñanza de matemáticas, video-encuesta, desempeño docente, evaluación docente

Triadic sequences (initiation-response-follow up) are a frequent type of interaction in classrooms in many different countries. This study describes, using a spoken discourse codification system, these sequences in 89 Chilean public middle-school level mathematics lessons, chosen at random from 684 classes that were filmed for a national evaluation. Triadic sequences were related to teachers' performance scores and lesson activities. The study identified a relatively shared interactional pattern, whose main features were: closed ended questions, low frequency of student participation and low use of metacognitive teacher follow-ups. In addition, when teaching mathematic skills, teachers generated more questions involving application of content, in comparison to other type of questions. Teachers that received a higher performance assessment produced a higher number of open-ended questions as well as a higher number of questions about students' personal experiences. The results of this study suggest that increasing the repertoire of teacher discourse is a potential area for teachers' training.

Keywords: discourse, teaching of mathematics, video-survey, teacher performance, teacher assessment

Darinka Radovic Sendra, Centro de Medición MIDE UC, Escuela de Psicología, Pontificia Universidad Católica de Chile, Santiago, Chile.

David Preiss Contreras, Escuela de Psicología, Pontificia Universidad Católica de Chile, Santiago, Chile.

El estudio fue realizado por la primera autora para cumplir con los requerimientos del Magíster en Psicología Educacional de la Pontificia Universidad Católica de Chile. El proyecto FONDECYT Nº 11060389 financió el trabajo de tesis de la primera autora. El trabajo del segundo autor contó, además, con el respaldo del Centro de Estudios de Políticas y Prácticas en Educación, Proyecto CIE01-CONICYT.

Se agradece, también, el apoyo de MIDE UC y de CPEIP, quienes dieron acceso al material incluido en el estudio.

La correspondencia relativa a este artículo debe ser dirigida a Darinka Radovic Sendra, Centro de Medición MIDE UC, Pontificia Universidad Católica de Chile, Avda. Vicuña Mackenna 4860, Macul, Santiago, Chile. E-mail: dradovic@uc.cl 
Investigar los procesos de enseñanzaaprendizaje, tal como ocurren dentro de las salas de clases, es útil para comprender los procesos pedagógicos que subyacen a los resultados de aprendizaje alcanzados por los estudiantes y evaluar el impacto de iniciativas de reforma educacional (Galton, Hargreaves, Comber, Wall \& Pell, 1999). La incorporación del video como herramienta de observación y registro sistemático de clases ha posibilitado la emergencia de iniciativas orientadas a desarrollar conocimiento relativo a estos procesos (Stigler, Gallimore \& Hiebert, 2000).

En Chile la implementación, a partir del año 2003, del Sistema Nacional de Evaluación del Desempeño Docente-DocenteMás-, ha favorecido el desarrollo de diversas iniciativas de observación y análisis de las dinámicas de enseñanza- aprendizaje en la salas de clases (Manzi \& Rosetti, 2004). En efecto, DocenteMás utiliza cuatro instrumentos de recolección de información, uno de los cuales corresponde a una clase video-grabada. A la fecha, este material audiovisual ha nutrido a una serie de iniciativas de investigación sobre la naturaleza de los procesos de enseñanza-aprendizaje (ver, por ejemplo, Araya \& Dartnell, 2009; González, Preiss \& San Martín, 2008; Milicic, Rosas, Scharager, García \& Godoy, 2008; Preiss, 2009, 2010). El presente estudio continuó esta línea de investigación, centrándose en la descripción de los patrones de discurso presentes en clases de matemáticas de segundo ciclo básico durante la ejecución de actividades públicas, esto es, que involucran la interacción profesor-curso de manera grupal.

El interés en Chile por el estudio del discurso en el aula matemática se origina en las falencias que muestran los estudiantes en su desempeño en esta área. La evidencia sugiere que las dinámicas en el aula juegan un papel relevante en los logros de los alumnos. Un análisis reciente de la evidencia de PISA 2006 en matemática, en el que se comparan los resultados de Chile con Polonia, España y Uruguay -países cultural y/o económicamente similares-, se encontró que la brecha de los resultados entre Chile y los dos países europeos se relacionaba con variables socioeconómicas del establecimiento y baja eficacia a nivel del aula. En otras palabras, lo que los chilenos ganaban por mayor escolaridad, lo perdían en términos relativos por su poca eficacia dentro de las salas de clases (Valenzuela, Bellei, Sevilla \& Oses, 2009).

\section{Las Secuencias Triádicas Como Objeto de Investigación}

Aprender matemáticas es participar de un proceso de socialización, iniciado en la escuela, sobre los modos de conocer usados por la comunidad de matemáticos y profesores de matemáticas. Según Nunes (2000), este proceso de socialización consiste en un proceso de redescripción representacional, en el que significados que se derivan de esquemas de acción relacionados con procesos matemáticos básicos (e.g., contar) se asocian a nuevos sistemas de signos. De acuerdo a Nunes (2000), estos procesos de redescripción -del esquema de acción al signo- son sociales en virtud de la naturaleza convencional de las matemáticas y su anclaje en procesos de interacción social. El trabajo de los profesores consiste, entonces, en promover este proceso de redescripción, favoreciendo la conexión entre las matemáticas intuitivas, que los estudiantes traen desde fuera de la escuela, y el manejo formal enseñado en el aula. Ben-Zvi y Sfard (2007) conciben el aprendizaje de las matemáticas justamente como el aprender a hablar de un modo nuevo; en particular, aprender a manejar nuevos tipos de discurso, tanto a nivel del objeto matemático como de metareglas. Desde esta perspectiva, para hacer más eficaz este aprendizaje, lo relevante no es solo hablar de las matemáticas sino cómo hablar acerca de estas. Para estudiar este proceso de re-descripción, en el presente estudio se pone atención al esqueleto del discurso docente: las secuencias triádicas.

Una gran cantidad de trabajo empírico ha mostrado que los intercambios entre profesores y estudiantes se organizan como secuencias triádicas iniciadas por los docentes, esto es, el profesor hace una pregunta, un estudiante es seleccionado para responderla y el profesor evalúa la respuesta (Mehan, 1979; Nassaji \& Wells, 2000; Sinclair \& Coulthard, 1975). Sinclair y Coulthard (1975) propusieron que el tercer movimiento de esta secuencia (evaluar 
la respuesta del alumno) podía responder a tres acciones distintas: aceptar/rechazar, evaluar y comentar. Esto llevó a que la secuencia triádica pasara a ser considerada como initiation-response-follow up o seguimiento (IRF por su sigla en inglés). El predominio de estas secuencias triádicas ha sido cuestionado porque podría desfavorecer a niños y niñas de culturas en las que este intercambio es poco común y, además, proveer pocas oportunidades para que los estudiantes expresen sus ideas o comenten las de otros (Wells \& Mejia Arauz, 2006). Sin embargo, se ha sugerido también que la secuencia triádica permite satisfacer las metas de escolarización y provee de un cierre a la interacción pedagógica (Newman, Griffin \& Cole, 1989): alguien que sabe (el conocedor primario) pide una respuesta, el conocedor secundario da la respuesta y el conocedor primario acepta la respuesta, dando el cierre necesario a la interacción (Berry, 1981).

Es importante destacar, sin embargo, que el foco de la investigación no debiera estar en el valor global de las secuencias triádicas, sino en las funciones que cada uno de los intercambios toma dentro del contexto de estas secuencias (Wells, 1999). Por ejemplo, si el profesor desea monitorear la comprensión de un concepto relevante previamente visto o revisar una tarea previamente realizada, es probable que la secuencia finalice con una evaluación del aporte del alumno, lo que no sucedería necesariamente cuando el tercer movimiento tiene como objetivo ampliar la respuesta del estudiante, extraer su significado o establecer conexiones con su experiencia (Wells, 1999).

Como un modo de estudiar la riqueza potencial de estos intercambios, Wells desarrolló un esquema de codificación en el que los movimientos discursivos son codificados en función de su propósito: las preguntas se diferencian dependiendo de si están dirigidas a obtener información previamente conocida, información personal o involucran una negociación de información. Respecto de los seguimientos, estos se pueden diferenciar entre aquellos orientados a evaluar, justificar, comentar o clarificar (Nassaji \& Wells, 2000). Este esquema de codificación ha sido utilizado en numerosas investigaciones sobre el discurso en la sala de clases (Nassaji
\& Wells, 2000; Wells, 1996, 1999; Wells \& Mejia Arauz, 2006), evidenciando que:

1. la elección de la pregunta inicial tiene una importante influencia en cómo la secuencia se desarrolla;

2. el seguimiento orientado a la evaluación de la respuesta del alumno tiende a suprimir la participación extendida de este; y

3. el seguimiento de evaluación puede llegar a generar interacciones similares a la conversación, si las preguntas exigen justificar, realizar conexiones o contraargumentar (Nassaji \& Wells, 2000).

Por último, existe evidencia que vincula la elección de preguntas y seguimientos que realiza el profesor y el rendimiento académico de los estudiantes. Por ejemplo, Nystrand $\mathrm{Wu}$, Gamoran, Zeiser y Long (2003) encontraron una relación directa entre el rendimiento de los estudiantes y la extensión del discurso más allá de la recitación, el uso de géneros de discurso que dan énfasis a las ideas de los estudiantes, la proporción de preguntas que no buscan información conocida, la proporción de preguntas que incorporan las respuestas anteriores de los estudiantes y el tiempo dedicado a la discusión.

\section{Discurso en la Sala y Patrones Instruccionales}

Una pregunta que se relaciona con los hallazgos anteriormente presentados es la relativa universalidad del tipo de interacciones y discursos dentro de las salas de clases. Los estudios de patrones de interacción en la sala de clases han mostrado comúnmente un desbalance en la cantidad de las contribuciones de profesores y estudiantes, baja reciprocidad en los intercambios y un desbalance en el control o poder en las relaciones (Burns \& Myhill, 2004; Newman et al., 1989). Por lo mismo, la investigación educacional ha descrito que en situaciones habituales de clase opera la regla de dos tercios: durante aproximadamente dos tercios del tiempo alguien habla, aproximadamente dos tercios del habla corresponde al profesor y aproximadamente dos tercios del habla del profesor consiste en preguntas y explicaciones (Mercer \& Littleton, 2007).

Si bien este desbalance en el discurso 
es universal, las video-encuestas del TIMSS revelaron que, aun cuando existen aspectos compartidos, es posible identificar la existencia de patrones instruccionales propios en cada país (Hiebert et al., 2003, 2005; Stigler et al., 2000; Stigler, Gonzales, Kawanaka, Knoll \& Serrano, 1999; Stigler \& Hiebert, 1999). Kawanaka y Stigler (1999), por ejemplo, mostraron que las clases de matemáticas alemanas, japonesas y estadounidenses tenían como rasgo común el predominio del profesor en el discurso existente en la clase y que el tipo de intercambio más común involucraba la entrega de información. En el caso de los estudiantes, se observó que los intercambios más recurrentes involucraban responder a preguntas. Sin embargo, los autores también constataron ciertas diferencias: los profesores alemanes realizaban casi en igual número intercambios orientados a entregar información y a suscitar respuestas de los estudiantes, mientras que los profesores japoneses y estadounidenses realizaban casi el doble de intercambios orientados a entregar información. Las preguntas orientadas a respuestas de alto orden (explicación y descripción) fueron más frecuentes entre profesores japoneses y las intervenciones de los estudiantes, más comunes en Alemania.

En Chile, Araya y Dartnell (2009) analizaron todos los videos de profesores de matemáticas de la ronda 2005 de DocenteMás. El 78,8\% de los videos analizados eran de profesores que hacían clases en el segundo ciclo de enseñanza básica y el grupo restante lo hacía en enseñanza media. Los autores mostraron que en todos los videos el proceso de enseñanza estaba centrado en el profesor, que es quien formula preguntas, expone en el pizarrón u organiza el trabajo individual de los estudiantes. Tal como también reportan Milicic et al. (2008), en los videos observados por Araya y Dartnell (2009) existe una dinámica claramente identificable incentivada por la estructura de la evaluación docente: los profesores marcan con claridad un inicio, segmentos intermedios y el final de la clase, distinguiéndose dos patrones típicos de comportamiento en los segmentos intermedios de la clase, uno que consiste en preguntar a los estudiantes y el otro, supervisar el trabajo, acercándose a ellos. Los autores reportan una baja incidencia de preguntas sobre matemáticas por parte de los estudiantes.

En otro estudio realizado en una muestra de 117 clases de matemáticas de segundo ciclo, Preiss (2010) mostró que el tipo de resolución de problemas favorecido en estas clases se basa en la práctica repetida, guiada por el profesor, de problemas concurrentes. Tal como en Estados Unidos, los profesores chilenos presentaban a los estudiantes definiciones y procedimientos que ellos debían practicar posteriormente. Por otro lado, la mayor parte del trabajo independiente observado involucraba trabajo individual en vez de trabajo grupal. Preiss (2010) describió estas clases como la apropiación privada de términos y procedimientos. En el dominio de lenguaje y comunicación, en el análisis de 128 clases, Preiss (2009) observó que, independientemente del nivel de competencia de los docentes, las preguntas y seguimientos de los profesores a las intervenciones de los estudiantes se orientaban a la regulación de la clase o al chequeo de información. Pese a esta estructura común, Preiss (2009) reportó la existencia de una correlación pequeña pero directa entre la evaluación de desempeño del profesor y la cantidad de preguntas y seguimientos que tenían mayor probabilidad de comprometer a los estudiantes en un pensamiento metacognitivo.

\section{Propósito del Estudio}

El propósito de esta investigación fue la descripción del discurso dentro de las salas de clases cuando la modalidad de trabajo es pública. Específicamente, se buscó describir cuáles son los tipos de preguntas o solicitudes que los profesores priorizan en la interacción con sus alumnos, con qué nivel de apertura los profesores tienden a plantear las preguntas a sus estudiantes, qué seguimientos de las intervenciones de los estudiantes son más frecuentes y qué tipo de intervenciones hacen los alumnos. Por otra parte, interesó explorar la relación entre el tipo de preguntas y seguimientos de los profesores y los tipos de actividades que realizan, y los distintos niveles de desempeño docente. 
Estudios previos sobre muestras similares (Preiss, 2009) sugieren que aquellos profesores identificados como destacados tienen un repertorio comunicativo relativamente más rico que sus pares en algunas medidas de metacognición. En el presente estudio se esperaba que estas diferencias se replicaran. Por lo tanto, la hipótesis del mismo fue que, en comparación con sus pares evaluados con un desempeño básico, profesores identificados como destacados realizan mayor número de:

1. preguntas con un nivel de desafío cognitivo más alto (solicitar la explicitación de un procedimiento para un problema matemático y solicitar una justificación para una regla general),

2. preguntas orientadas a relacionar la experiencia personal de los estudiantes con los contenidos matemáticos revisados,

3. preguntas abiertas con más de una posibilidad de respuesta, y

4. seguimientos orientados a reformular las respuestas de los estudiantes.

El estudio esperaba que esta diferencia también fuera significativa entre profesores destacados y competentes.

\section{Método}

\section{Participantes}

El presente estudio basa sus resultados en el análisis de videos de clases realizadas por profesores (una clase por profesor) de matemática de segundo ciclo básico de establecimientos de educación municipal que participaron en el proceso nacional obligatorio de la evaluación docente en el año 2005. Del total de 684 profesores de matemática de segundo ciclo que participaron de esta evaluación y que permitieron el uso de los videos para fines de investigación, se seleccionaron de manera aleatoria 89 videos. Aun cuando el proceso de selección fue aleatorio, la inspección de la composición sociodemográfica de la muestra reveló que los profesores que trabajan en escuelas rurales $(24,7 \%)$ y los de sexo masculino $(45 \%)$ estaban sobre-representados en relación al porcentaje nacional de profesores trabajando en escuelas municipales el año 2005, los cuales correspondían a un $20 \%$ y a un $30 \%$, respectivamente (Chile, Ministerio de Educación, 2006).

Además de los videos, se contó con los resultados de la evaluación de los profesores en el proceso llevado a cabo ese año, tanto respecto de la categoría final, que considera la evaluación recibida en el portafolio, la evaluación de pares, la evaluación del supervisor y una autoevaluación, así como la categoría obtenida considerando solo el portafolio. Las categorías incluyen cuatro niveles de desempeño: (a) insatisfactorio, categoría con la que el profesor no aprueba la evaluación docente; (b) básico; (c) competente y (d) destacado. En la Tabla 1 se presenta la distribución de los profesores de la muestra según estas categorías de evaluación.

Tabla 1

Distribución de Profesores de la Muestra Según Nivel de Desempeño en la Evaluación Docente

\begin{tabular}{lcccc}
\hline \multirow{2}{*}{$\begin{array}{c}\text { Nivel de } \\
\text { desempeño }\end{array}$} & \multicolumn{2}{c}{ Categoría final } & \multicolumn{2}{c}{ Categoría portafolio } \\
\cline { 2 - 5 } & Frecuencia & Porcentaje & Frecuencia & Porcentaje \\
\hline Insatisfactorio & 2 & 2,25 & 4 & 4,49 \\
Básico & 12 & 13,48 & 40 & 44,94 \\
Competente & 57 & 64,04 & 43 & 48,31 \\
Destacado & 18 & 20,22 & 2 & 2,25 \\
\hline
\end{tabular}




\section{Instrumento}

Una pauta guió el proceso de codificación del discurso, siendo su objetivo la identificación de la principal función y tipo de cada intercambio orientado a la interacción profesor-alumno. Esta pauta fue construida en base a otros esquemas de codificación (Hiebert et al., 2003; Preiss, 2009, 2010; Stigler et al., 1999; Wells, 1999). En esta pauta se distinguieron intercambios con tres funciones prioritarias:

1. solicitud del profesor de participación de los estudiantes,

2. intervenciones de los estudiantes y

3. seguimientos del profesor de las intervenciones de los estudiantes.

Para las solicitudes del profesor se identificó si estas tenían como principal función el control del flujo de la clase y la generación de participación de los estudiantes (intervenciones no orientadas prioritariamente a recoger información de contenidos, denominados control) o si estas buscaban obtener información matemática. Para los intercambios con esta última función se procuró identificar distintos objetivos de aprendizaje, utilizando lineamientos basados en la taxonomía desarrollada por Bloom (Anderson, Krathwohl, Airasian \& Bloom, 2001), especificándose solicitudes de:

1. experiencia personal relacionada con los contenidos matemáticos trabajados,

2. definición de conceptos matemáticos (recordar),

3. ejecución de un procedimiento matemático enunciado por el profesor (ejercitar),

4. aplicación de un concepto o procedimiento matemático a una situación matemática o no matemática (aplicar),

5. explicitación de un procedimiento requerido o realizado para la resolución de un problema (metacognición) y

6. desarrollo de la justificación de una regla general o principio matemático.

En la Tabla 2 se presentan ejemplos prototípicos de cada una de las solicitudes de información relacionadas con contenidos matemáticos identificados.

Además de identificar el tipo de intercambio, en las solicitudes de información del profesor a sus estudiantes se evaluó el nivel de apertura de cada una de ellas, con- siderándose si estas consistían en:

1. preguntas cerradas que solicitaban al alumno respuestas sí/no o elegir una alternativa de entre varias;

2. preguntas que buscaban solo una respuesta correcta que, con la información entregada por el profesor, el alumno no podía derivar por azar, y

3. preguntas con más de una respuesta posible. En la Tabla 3 se muestran ejemplos de estos códigos.

Respecto de las intervenciones del alumno, se distinguió si estas correspondían a una respuesta a alguna pregunta del profesor o si eran intervenciones de solicitud de atención del profesor o de otro alumno, si era una pregunta espontánea o si correspondía a la corrección de una información previamente dicha.

Por último, en relación a los seguimientos por parte del profesor de las intervenciones de los estudiantes, se distinguieron monosílabos, repeticiones que el profesor realiza de la respuesta de los alumnos, evaluaciones y reformulaciones. Estas últimas consisten en que el profesor considera una intervención del alumno e incorpora información nueva o la reformula a un lenguaje matemático. Cada una de las intervenciones identificadas fue marcada, lo que permitió posteriormente contabilizar la frecuencia de cada una de ellas por clase y por segmento.

\section{Procedimiento}

Los videos forman parte de un portafolio que el profesor debe entregar para participar del proceso de evaluación docente y el uso de esta información para fines de investigación debe ser autorizada por este, firmando un consentimiento informado. En el presente estudio se incluyeron solo a profesores que autorizaron el uso de la información para estos fines.

La filmación de los videos se realizó de acuerdo a un procedimiento estandarizado, descrito en detalle en Preiss (2009).

\section{Análisis de Datos}

Cada clase fue transcrita en su totalidad, identificándose los distintos turnos de habla, correspondientes a las distintas 
Tabla 2

Ejemplos de Preguntas de Información de Contenidos Matemáticos

\begin{tabular}{|c|c|}
\hline Tipo de pregunta & Ejemplos \\
\hline Experiencia personal & $\begin{array}{l}\text { ¿En qué región vivimos nosotros? } \\
\text { ¿Qué más venden en las fondas? }\end{array}$ \\
\hline Avance en la tarea & $\begin{array}{l}\text { ¿Terminaron? } \\
\text { ¿Quién hizo la número } 2 ?\end{array}$ \\
\hline Concepto & $\begin{array}{l}\text { ¿Cuáles son los triángulos de tres lados iguales? } \\
\text { ¿Cómo se llama el número de abajo de la fracción? }\end{array}$ \\
\hline Solución & $\begin{array}{l}\text { Y quince dividido en tres es: } \\
\text { Tres más } 5 \text { es } 8 \text { y } 8 \text { por } 8 \text {, sería: }\end{array}$ \\
\hline Aplicación & $\begin{array}{l}\text { ¿Quién da un ejemplo de magnitudes directamente proporcionales? } \\
\text { ¿Qué nota tendría que sacarse Andrea para tener un promedio } 6,5 ?\end{array}$ \\
\hline Procedimiento & $\begin{array}{l}\text { ¿Cómo calculamos la nota que tenía que sacarse Andrea para terminar con } \\
\text { un } 6,5 ?\end{array}$ \\
\hline Justificación & $\begin{array}{l}\text { ¿Por qué se llamarán enteros? } \\
\text { ¿Por qué creen ustedes que la balanza está desequilibrada? }\end{array}$ \\
\hline
\end{tabular}

Tabla 3

Ejemplos de Nivel de Apertura de Preguntas de Información de Contenidos Matemáticos

\begin{tabular}{|c|c|}
\hline Nivel de apertura & Ejemplos \\
\hline Pregunta cerrada & $\begin{array}{l}15+8 \text { es } 23, \text { ¿está eso correcto? } \\
\text { ¿Cuál es el triángulo isósceles, el de tres o dos lados iguales? }\end{array}$ \\
\hline $\begin{array}{l}\text { Pregunta abierta } 1 \text { respuesta } \\
\text { correcta }\end{array}$ & $\begin{array}{l}\text { Entonces } 15+8 \text { es... } \\
\text { ¿Cuál es el triángulo de } 2 \text { lados iguales? }\end{array}$ \\
\hline $\begin{array}{l}\text { Pregunta abierta más de } 1 \\
\text { respuesta correcta }\end{array}$ & $\begin{array}{l}\text { ¿De qué distintas maneras podemos reconocer a un triángulo } \\
\text { isósceles? }\end{array}$ \\
\hline
\end{tabular}

interacciones del profesor o los alumnos, especificándose al hablante de cada uno de ellos. Para cada uno de los turnos de habla se contó, además, con información recogida en un estudio anterior con los mismos videos (Preiss, 2010), en el que se segmentó la clase de acuerdo a la modalidad de interacción y al objetivo prioritario de la actividad realizada. Respecto de la modalidad de interacción, se distinguieron segmentos con discurso prioritariamente público o privado. En cuanto al objetivo de la actividad, se distinguieron segmentos dedicados a la introducción de contenidos, la práctica de contenidos, la revisión de contenidos, a tra- bajo no matemático, a la identificación de las metas de la clase (objetivo) o a la síntesis de las ideas claves trabajadas durante la clase. Aproximadamente un tercio de las clases analizadas fueron doble-codificadas, presentando cada uno de los códigos un nivel de acuerdo aceptable $(p>0,75$, Preiss, 2010). Al realizar la transcripción del discurso hablado, a cada turno de habla se le asignó un identificador que permitió posteriormente relacionarlo con la modalidad de interacción en la que este se desarrolló y el tipo de actividad en curso.

El proceso de codificación fue realizado por dos codificadoras y la primera autora, 
durante dos meses de trabajo, incluyendo capacitación con reuniones de revisión de pauta, revisión de clases doblemente codificadas y entrega de retroalimentación escrita con ejemplos, según errores comunes de cada una de las codificadoras. En cada una de las clases doble-codificadas se identificaron los acuerdos (el mismo código asignado al mismo turno de habla) y desacuerdos para cada uno de los códigos asignados a los segmentos analizados y se calculó el porcentaje de acuerdo sobre el total de códigos de la clase para cada una de las áreas descritas (preguntas del profesor, intervenciones del alumno y aceptaciones del profesor). El periodo de calibración fue llevado a cabo hasta que las codificadoras mostraron un nivel de acuerdo sostenido (al menos 5 clases consecutivas), sobre el $65 \%$ en cada uno de los aspectos evaluados en la pauta. Este porcentaje fue definido en base a la convención tácita de que el $65 \%$ de acuerdo corresponde al estándar mínimo aceptable (Tinsley \& Brown, 2000). Una vez que se alcanzó el estándar definido en todas las áreas evaluadas, se detuvo la doble codificación, doble codificándose cinco clases de las restantes para monitorear la mantención de este nivel de acuerdo. Los desacuerdos fueron dirimidos según la corrección de la primera autora.

Como indicador final de concordancia entre los jueces se calcularon correlaciones bivariadas de Pearson entre las frecuencias de cada tipo de intercambio en cada una de las clases analizadas por cada una de las codificadoras. Todas estas mostraron un $r(30)>0,70, p<0,001$, excepto en el nivel de apertura de más de una respuesta posible $(r[30]=0,69, p<0,001)$, seguimiento de reformulación $(r[30]=0,61, p<0,001)$ e intervención del alumno en que pide corregir $(r[30]=0,65, p<0,001)$, considerándose de todas formas aceptables.

Para el análisis de la información se calcularon las frecuencias de cada clase de cada uno de los códigos incorporados. Se realizaron análisis descriptivos de estas frecuencias de aparición en la muestra de clases completas, tanto porcentajes de cada código respecto del tipo de intervención analizada como promedios del total de intercambios identificados en las clases analizadas.
Para evaluar la relativa estabilidad del tipo de preguntas en una misma clase, pero en el marco de distintas actividades, se calcularon las frecuencias de estos intercambios en cada una de las actividades identificadas. Debido a que los profesores dedican tiempos muy disímiles a las distintas actividades, el análisis se realizó considerando el número de intercambios por minuto para cada una de las preguntas identificadas. Interesó también observar si los profesores formulaban distinta cantidad de preguntas abiertas con más de una posibilidad de respuesta según el objetivo de la actividad en desarrollo, para lo que se realizó un ANOVA de medidas repetidas, utilizando como factor intra-sujeto la cantidad de este tipo de preguntas en cada una de las cinco actividades identificadas, reportándose la prueba ajustada con el criterio de Greenhouse-Geisser.

Como medida de asociación entre el tipo de preguntas y seguimientos realizados por el profesor y su evaluación de desempeño docente se utilizó el coeficiente de correlación lineal de Pearson de dos colas entre la frecuencia de aparición de cada intercambio realizado por el profesor y el puntaje final asignado al portafolio de la evaluación docente. Para evaluar las diferencias por nivel de desempeño docente se utilizó la categoría final de la evaluación, sin considerar a los profesores evaluados como insatisfactorios (ya que correspondían a solo dos profesores). De este modo, se compararon tres grupos (profesores con evaluación básica, competente y destacada). Por medio de múltiples ANOVAs de un factor se evaluaron las diferencias en: (a) la cantidad de preguntas orientadas a solicitar la explicitación de un procedimiento para un problema matemático específico, (b) solicitud de una justificación para una regla general, (c) preguntas orientadas a relacionar la experiencia personal de los estudiantes con contenidos matemáticos, (d) preguntas abiertas con más de una posibilidad de respuesta y (e) uso de seguimientos orientados a reformular las respuestas de los estudiantes. Para los modelos significativos se realizaron contrastes planificados, comparando con $t$ de Student de una cola los promedios de los profesores de nivel de desempeño destacado con los de sus pares 
evaluados como básicos y con los evaluados como competentes.

Por último, como medidas de tamaño del efecto se reportan los eta al cuadrado $\left(\eta^{2}\right)$ para los modelos generales significativos y los $d$ de Cohen para los pares contrastados.

\section{Resultados}

\section{Resultados Descriptivos}

Durante las secuencias de interacción pública, las preguntas o solicitudes de participación a los estudiantes son un mecanismo frecuentemente utilizado, observándose en promedio 3,1 preguntas por minuto $(D E$ $=1,1)$. De estas, gran parte corresponde a preguntas orientadas al control del flujo de la clase, ya sea para verificar la atención del alumno o para la entrega de turnos, observándose en promedio que, del total de preguntas realizadas en cada clase, el 49\% (DE $=15,3$ ) cumple esta función. Además, este tipo de solicitudes son las que aparecen en promedio una mayor cantidad de veces, $M$ $=33, D E=17,9$.

De las preguntas orientadas a obtener información de los estudiantes respecto de los contenidos de la clase, se detectó que las mayormente formuladas corresponden a preguntas de conceptos y aplicaciones, observándose en cada clase un promedio 14
$(D E=12,5)$ preguntas referidas a conceptos y $11,6(D E=10,0)$ aplicaciones de conceptos o procedimientos matemáticos a situaciones matemáticas y no matemáticas. Ambas tienen también una alta representación en las clases analizadas (las de conceptos aparecen al menos una vez en el $94 \%$ de las clases y las de procedimientos, en un $96 \%$ de las clases). El resto de las preguntas que buscan obtener información relacionada con los contenidos de la clase aparece con una frecuencia bastante más baja. En cerca del $70 \%$ de las clases los profesores realizan al menos una pregunta que solicita a los estudiantes explicitar el procedimiento requerido para resolver un problema matemático y en cerca de un $50 \%$, preguntas orientadas a justificar una regla general, pedir experiencia personal de los estudiantes o solucionar una operación matemática explicitada por el profesor. Las frecuencias de estas preguntas también resultaron ser más bajas, observándose los promedios de aparición que se detallan en la Tabla 4.

Respecto del nivel de apertura de las preguntas, se observó que las preguntas con más de una respuesta correcta aparecieron al menos una vez en solo el $43 \%$ de las clases, mientras que las preguntas planteadas de forma abierta con solo una posibilidad de respuesta aparecieron en el 100\% de las clases analizadas, más comunes que las que se planteaban en base a respuestas del tipo síl

Tabla 4

Promedio de Preguntas por Clase Según Tipo de Pregunta

\begin{tabular}{lcc}
\hline \multicolumn{1}{c}{ Tipo de pregunta } & Promedio & Desviación estándar \\
\hline Control & 33,00 & 17,90 \\
Concepto/definición & 13,98 & 12,54 \\
Aplicación & 11,64 & 10,03 \\
Solución & 3,26 & 5,32 \\
Procedimiento & 2,81 & 3,41 \\
Experiencia personal & 1,89 & 3,02 \\
Justificación & 1,48 & 2,19 \\
\hline
\end{tabular}


no o con alternativas explicitadas, que aparecen en un $87 \%$ de las clases. En la Tabla 5 se presentan los promedios de aparición de las preguntas para los distintos niveles de apertura identificados.

En lo que dice relación con las intervenciones de los estudiantes, del total de turnos de habla de los estudiantes, un 95\% corresponde a respuestas a solicitudes o preguntas de los profesores y solo el 1\%, a preguntas espontáneas de los estudiantes. La extensión de las intervenciones de los estudiantes, medida en base al total de palabras, tiende a ser corta: un $70 \%$ corresponde a intervenciones de una o dos palabras y solo un $11 \%$, a intervenciones de más de seis palabras.

Por último, se observó que los seguimientos que realiza el profesor de las intervenciones de los estudiantes aparecen al menos una vez en la mayoría de las clases (sobre el 90\% de las clases). Sin embargo, difieren ampliamente en la cantidad de seguimientos por tipo. En la Tabla 6 se presentan los promedios de los distintos seguimientos identificados.

\section{Diferencias en las Preguntas Realizadas en Actividades con Distintos Propósitos}

Para analizar la relativa estabilidad del tipo de preguntas dentro de una misma clase, pero en el marco de distintas actividades, se observaron las frecuencias por minuto de estos intercambios en cada una de las actividades identificadas. Los profesores realizan una cantidad mayor de preguntas, tanto de control del flujo de la clase como de contenidos propiamente tales, durante actividades de práctica de contenidos, las que en promedio alcanzan las 8,8 preguntas por minuto $(D E=6,6)$. Durante el resto de las actividades, el número de preguntas por minuto no alcanza a un promedio de tres: $2,9(D E=3,5)$ en actividades de introducción de contenidos, $2,5(D E=3,3)$ en actividades de revisión de contenidos, 1,7 ( $D E=$ $2,4)$ en momentos de la clase dedicados a la explicitación de metas y 2,2 $(D E=2,9)$ en actividades dedicadas a sintetizar los contenidos trabajados en la clase. Lo anterior da cuenta de que los profesores tienden a realizar menor número de preguntas cuan-

Tabla 5

Promedio de Preguntas por Clase Según Nivel de Apertura de la Pregunta

\begin{tabular}{lcc}
\hline \multicolumn{1}{c}{ Nivel de apertura } & Promedio & Desviación estándar \\
\hline $\begin{array}{l}\text { Pregunta cerrada } \\
\text { Abierta con solo una } \\
\text { respuesta }\end{array}$ & 3,98 & 4,43 \\
$\begin{array}{l}\text { Abierta con más de una } \\
\text { respuesta }\end{array}$ & 30,63 & 17,55 \\
\hline
\end{tabular}

Tabla 6

Promedio de Seguimientos por Clase Según Tipo

\begin{tabular}{lcc}
\hline \multicolumn{1}{c}{ Tipo de seguimiento } & Promedio & Desviación estándar \\
\hline Monosílabo & 8,16 & 6,60 \\
Repetición & 21,87 & 11,55 \\
Evaluación & 10,80 & 8,84 \\
Reformulación & 4,63 & 3,29 \\
\hline
\end{tabular}


do están introduciendo los objetivos de la clase (metas) o cuando están realizando un cierre de la misma (síntesis), que cuando están trabajando los contenidos propiamente tales. La distribución de frecuencias de los distintos tipos de preguntas de contenidos por minuto, según el objetivo de la actividad en desarrollo, se presenta en la Figura 1.

Como se observa en la Figura 1, durante todas las actividades identificadas, excepto durante las actividades de práctica de contenidos, se mantiene el patrón observado en la clase completa de un predominio de preguntas referidas a conceptos. Cuando los profesores realizan actividades orientadas a practicar contenidos matemáticos, coincidente con el objetivo de la actividad, realizan una mayor cantidad de preguntas de aplicación.

Respecto de las diferencias en la cantidad de preguntas abiertas con más de una posibilidad de respuesta según el objetivo de la actividad en desarrollo, el ANOVA de medidas repetidas, utilizando la frecuencia de este tipo de preguntas para cada una de las cinco actividades identificadas como factor intrasujeto, mostró que no existen diferencias significativas, $F(3,2,278,4)=0,82, p=0,49$.

\section{Diferencias en el Patrón de Discurso Según Evaluación de Desempeño Docente}

Solo la cantidad de preguntas referidas a justificaciones de reglas generales y la cantidad de preguntas abiertas con más de una posibilidad de respuesta mostraron una correlación directa significativa, aunque de magnitud pequeña, con el puntaje obtenido por los profesores en el portafolio de la evaluación docente: $r(89)=0,31, p=0,003 \mathrm{y}$ $r(89)=0,23, p=0,03$, respectivamente.

$\mathrm{Al}$ evaluar las diferencias de los tipos de intercambios priorizados por los profesores según su nivel de desempeño final en la evaluación, se encontraron diferencias significativas respecto de la cantidad de preguntas de experiencia personal, $F(2,84)=$ $3,62 p=0,03, \eta^{2}=0,08$, y la cantidad de preguntas abiertas, $F(2,84)=6,48, p=0,002$, $\eta^{2}=0,13$. No se encontraron diferencias en la cantidad de preguntas de procedimientos, $F(2,84)=0,36, p=0,73$, en la solicitud de justificaciones, $F(2,84)=2,26, p=0,11$, ni en el uso de seguimientos con reformulación de las respuestas de los estudiantes, $F(2,84)=2,80, p=0,76$.

Como se observa en la Tabla 7 , tanto los promedios de preguntas de experiencias personales de los estudiantes relacionadas con contenidos matemáticos como los promedios de preguntas de mayor nivel de apertura son mayores en el grupo de profesores de desempeño destacado. Al evaluar las diferencias a través de contrastes planificados, se encontraron diferencias significativas en todos los pares contrastados respecto del nivel de apertura de la pregunta: básico-destacado $t(84)=-2,88, p<0,01, d=$ 0,85 y competente-destacado $t(84)=-3,39, p$ $<0,01, d=0,70$. En el caso de las pregun-

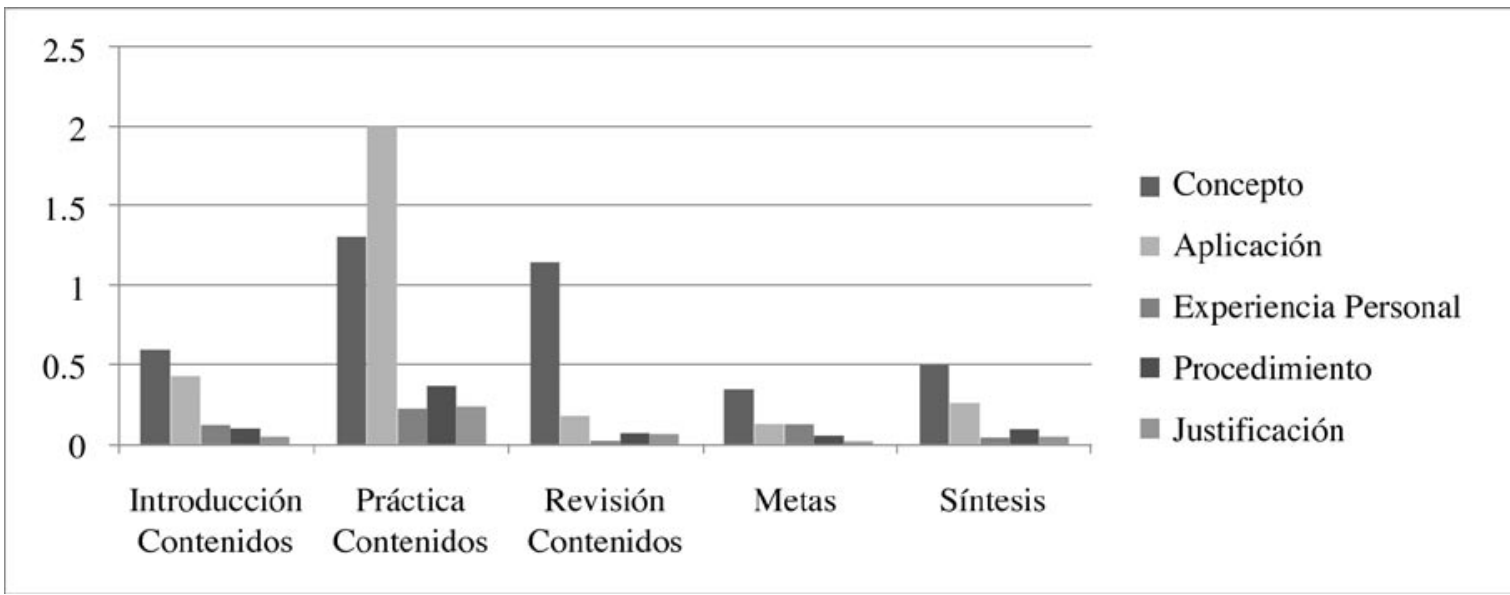

Figura 1. Frecuencias por minuto de preguntas según actividad. 
Tabla 7

$F$ de Levene, Promedio y Desviación Estándar de Preguntas y Seguimientos, e Intervalos de Confianza de las Diferencias con el Nivel de Desempeño Docente Destacado

\begin{tabular}{|c|c|c|c|c|c|c|c|c|c|}
\hline \multirow{3}{*}{ Tipos de preguntas } & \multirow{3}{*}{$F$ Levene } & \multicolumn{8}{|c|}{ Nivel de desempeño docente } \\
\hline & & \multicolumn{3}{|c|}{$\begin{array}{l}\text { Básico } \\
(n=12)\end{array}$} & \multicolumn{3}{|c|}{$\begin{array}{c}\text { Competente } \\
(n=57)\end{array}$} & \multicolumn{2}{|c|}{$\begin{array}{c}\text { Destacado } \\
(n=18)\end{array}$} \\
\hline & & $M$ & $D E$ & $\begin{array}{c}95 \% \text { IC } \\
\text { diferencia } \\
\text { con media } \\
\text { destacados }\end{array}$ & $M$ & $D E$ & $\begin{array}{c}95 \% \text { IC } \\
\text { diferencia } \\
\text { con media } \\
\text { destacados }\end{array}$ & $M$ & $D E$ \\
\hline Procedimientos & $\begin{array}{c}1,51 \\
p=0,23\end{array}$ & 3,17 & 4,20 & & 2,92 & 3,55 & & 2,22 & 2,51 \\
\hline Justificaciones & $\begin{array}{c}2,85 \\
p=0,06\end{array}$ & 0,50 & 0,80 & & 1,51 & 2,32 & & 2,22 & 2,29 \\
\hline $\begin{array}{l}\text { Seguimientos con } \\
\text { reformulación }\end{array}$ & $\begin{array}{c}0,73 \\
p=0,49\end{array}$ & 5,33 & 2,93 & & 4,54 & 3,26 & & 4,72 & 3,79 \\
\hline $\begin{array}{l}\text { Experiencia } \\
\text { personal }\end{array}$ & $\begin{array}{c}1,23 \\
p=0,30\end{array}$ & 1,92 & 3,09 & $-3,83,0,55$ & 1,40 & 2,69 & $-3,74,0,56$ & 3,56 & 3,65 \\
\hline $\begin{array}{l}\text { Preguntas de más } \\
\text { de una respuesta } \\
\text { posible }\end{array}$ & $\begin{array}{c}9,99 \\
p<0,001\end{array}$ & 0,42 & 0,79 & $-2,49,-0,46$ & 0,63 & 1,05 & $-1,20,-0,52$ & 1,89 & 2,30 \\
\hline
\end{tabular}

tas de experiencias personales de los estudiantes, solo fueron significativas las diferencias entre los profesores competentes y destacados: $t(84)=-2,69, p=0,01, d=0,67$. Para este último tipo de preguntas los profesores evaluados con un desempeño básico no mostraron diferencias con los profesores destacados: $t(84)=-1,49, p=0,07$.

\section{Conclusiones}

El presente estudio confirmó, en una muestra de profesores de escuelas municipales del sistema educacional chileno, los hallazgos de otras investigaciones locales respecto de: (a) la mayor frecuencia de preguntas orientadas al control del flujo de la clase más que al desarrollo de la comprensión de contenidos matemáticos; (b) el predominio de preguntas de bajo desafío cognitivo, orientadas a monitorear el recuerdo de conceptos y la aplicación a situaciones sencillas; (c) la baja apertura de estas preguntas, lo que potencialmente genera (d) una menor participación de los estudiantes en los procesos de construcción del conocimien- to y (e) el predominio de formas de seguimiento de las respuestas de los estudiantes basadas en la repetición y evaluación de las mismas.

Respecto de la organización del discurso en los distintos momentos de la clase, se observó que los profesores tienden a aumentar el número de intercambios orientados a la generación de participación de los estudiantes cuando realizan actividades cuyo principal propósito es la práctica compartida de contenidos matemáticos. En este tipo de actividades se observó también que los profesores priorizan preguntas de aplicación por sobre la realización de preguntas orientadas al monitoreo del aprendizaje de conceptos, las que son más comunes en las clases en general y en todas las otras actividades identificadas.

Por último, este patrón discursivo tiende a ser relativamente homogéneo y presentarse en formas similares en clases dirigidas por profesores evaluados con distinto nivel de desempeño, aunque se observa una leve tendencia a la mayor realización de preguntas abiertas con más de una posibilidad de respuesta y preguntas de jus- 
tificación en profesores con un mayor nivel de desempeño docente. Este patrón es bastante similar al observado previamente en clases de lenguaje y comunicación (Preiss 2009), lo cual insinúa que este patrón discursivo trasciende dominios. La homogeneidad del patrón discursivo sugiere que estos hallazgos describen modos relativamente típicos de hablar y comunicar en las aulas de la educación pública chilena, compartidos además con modos de hablar típicos de otros países. Una explicación de este isomorfismo mencionada por Givvin, Hiebert, Jacobs, Hollingsworth \& Gallimore (2005) es que actualmente existen en la mayoría de los establecimientos educacionales ciertos elementos universales que dan forma a las prácticas de los profesores, pudiendo enumerarse el ambiente físico de las salas de clases, las dinámicas sociales que ahí se dan y los contenidos que deben ser enseñados y aprendidos. Ya sea que estas comunalidades sean culturales (propias de Chile) o universales (compartidas en distintas partes del mundo), con base en los hallazgos de este estudio es posible afirmar que, al menos en las salas de clases del sistema público chileno, parecieran haber más semejanzas que diferencias en términos del discurso del profesor cuando se dirige a todos los alumnos de la sala.

Estos resultados, sin embargo, son preliminares y deben ser entendidos considerando las principales limitaciones del estudio. En primer lugar, ellos corresponden a clases dictadas con propósitos de una evaluación de desempeño y, por lo mismo, pueden no ser enteramente representativas de aquellas realizadas en un contexto natural. Aun así, es altamente probable que en sus elementos substantivos estas clases reflejen tendencias generales. El proceso instruccional es altamente estructurado y los profesores suelen reposar en sus modos habituales de enseñar para ejecutar su actividad, aun cuando esta actividad esté interferida por la observación de externos o por el uso de una cámara de video (Stigler et al., 2000). En segundo lugar, la muestra incluye un número menor de profesores de desempeño destacado. Futuros estudios debieran considerar estrategias de muestreo estratificado para evaluar más adecuadamente diferen- cias entre profesores de distintos niveles de desempeño.

El estudio sugiere que una potencial área de capacitación de profesores es el aumento del repertorio de su discurso. Dado que el repertorio de preguntas está restringido a la solicitud de conceptos y aplicaciones, la gran mayoría de las intervenciones de los estudiantes son respuestas a estas preguntas, lo cual limita sus aproximaciones a los contenidos de matemática. Esta conclusión se basa solo en la información levantada del discurso público en la sala y obvia la posibilidad de que las actividades desarrolladas en forma privada, o como tareas para desarrollar fuera de clase, ofrezcan a los estudiantes oportunidades desafiantes y problemas que impliquen, por ejemplo, derivar reglas generales o explicitar procedimientos. No obstante, parece claro que, cuando los profesores orientan la actividad a la generación de un discurso común, se sienten más cómodos en los ámbitos más básicos de la jerarquía de dominios cognitivos, esto es, reconocer y comprender (Anderson et al., 2001). Estos resultados son también compatibles con aquellos reportados por Araya y Dartnell (2009), quienes indicaron que no se observan demostraciones matemáticas en los videos analizados. Al parecer, los profesores estudiados priorizan el control de la clase y las respuestas conocidas por sobre la exploración de conocimientos, valorando la cantidad (mayor cantidad de interacciones simples) por sobre la calidad (interacciones más profundas).

Un modo alternativo de promover un cambio en el discurso de los profesores es hacerlo mediante una transformación de los textos de apoyo que estos usan, en los cuales sería conveniente incluir estrategias estructuradas de enseñanza que descansen en preguntas abiertas y en un diálogo más colaborativo con los estudiantes. La evidencia muestra, sin embargo, que los profesores escasamente usan textos (Araya \& Dartnell, 2009) en sus clases y que tienden a descansar en el uso de guías para la práctica repetida de habilidades (Preiss, 2010). Por lo tanto, para que esta estrategia tuviese éxito es necesario reinstalar el valor de los textos de apoyo en la implementación de las actividades docentes. 


\section{Referencias}

Anderson, L. W., Krathwohl, D. R., Airasian, P. W. \& Bloom, B. S. (2001). A taxonomy for learning, teaching, and assessing: A revision of Bloom's taxonomy of educational objectives. New York, NY: Longman.

Araya, R. \& Dartnell, P. (2009). Saber pedagógico y conocimiento de la disciplina matemática en docentes de educación general básica y media. En Chile, Ministerio de Educación (Ed.), Selección de investigaciones primer concurso FONIDE: evidencias para políticas públicas en educación (pp. 157-198). Santiago, Chile: Editor, Departamento de Estudios y Desarrollo.

Ben-Zvi, D. \& Sfard, A. (2007). Ariadne's thread, Daedalus' wings, and the learner's autonomy. Education \& Didactique, 1(3), 117-134.

Berry, M. (1981). Systemic linguistics and discourse analysis: a multi-layered approach to exchange structure. En M. Coulthard \& M. Montgomery (Eds.), Studies in discourse analysis (pp. 120145). London, Reino Unido: Routledge/Thoemms.

Burns, C. \& Myhill, D. (2004). Interactive or inactive? A consideration of the nature of interaction in whole class teaching. Cambridge Journal of Education, 34, 35-49. doi:10.1080/0305764042000183115

Chile, Ministerio de Educación (2006). Estadísticas de la educación 2005. Santiago, Chile: Autor, Departamento de Estudios y Desarrollo.

Galton, M., Hargreaves, L., Comber, C., Wall, D. \& Pell, T. (1999). Changes in patterns of teacher interaction in primary classrooms: 1976-96. British Educational Research Journal, 25, 23-37. doi:10.1080/0141192990250103

Givvin, K. B., Hiebert, J., Jacobs, J. K., Hollingsworth, H. \& Gallimore, R. (2005). Are there national patterns of teaching? Evidence from the TIMSS 1999 video study. Comparative Education Review, 49, 311-343. doi:10.1086/430260

González, J., Preiss, D. \& San Martín, E. (2008). Evaluando el discurso docente: desarrollo de un modelo Rasch a partir de evidencia audiovisual de profesores chilenos de primer ciclo de educación básica en el área de lenguaje. Revista Iberoamericana de Evaluación Educativa, 1(2), 137-147.

Hiebert, J., Gallimore, R., Garnier, H., Givvin, K. B., Hollingsworth, H., Jacobs, J. ... Stigler, J. (2003). Teaching mathematics in seven countries: Results from the TIMSS 1999 video study. Washington, DC: U.S. Department of Education, National Center for Education Statistics.

Hiebert, J., Stigler, J. W., Jacobs, J. K., Givvin, K. B., Garnier, H., Smith, M. ... Gallimore, R. (2005). Mathematics teaching in the United States today (and tomorrow): Results from the TIMSS 1999 video study. Educational Evaluation and Policy Analysis, 27, 111-132. doi:10.3102/016237370270 02111

Kawanaka, T. \& Stigler, J. W. (1999). Teachers' use of questions in eighth-grade mathematics classrooms in Germany, Japan, and the United States. Mathematical Thinking and Learning, 1, 255-278. doi: 10.1207/s15327833mt10104_1
Manzi, J. \& Rosetti, J. (2004). La evaluación educacional en Chile: antecedentes y desarrollo reciente. Persona y Sociedad, 18(3), 221-242.

Mehan, H. (1979). Learning lessons: Socialorganization in the classroom. Cambridge, MA: Harvard University Press.

Mercer, N. \& Littleton, K. (2007). Dialogue and the development of children's thinking: A sociocultural approach. New York, NY: Routledge.

Milicic, N., Rosas, R., Scharager, J., García, M. R. \& Godoy, C. (2008). Diseño, construcción y evaluación de una pauta de observación de videos para evaluar calidad del desempeño docente. Psykhe, 17(2), 79-90. doi:10.4067/S071822282008000200007

Nassaji, H. \& Wells, G. (2000). What's the use of 'triadic dialogue'?: An investigation of teacherstudent interaction. Applied Linguistics, 21, 376406. doi:10.1093/applin/21.3.376

Newman, D., Griffin, P. \& Cole, M. (1989). The construction zone: Working for cognitive change in school. Cambridge, MA: Cambridge University Press.

Nunes, T. (2000). Mathematics learning as the socialization of the mind. Mind, Culture, and Activity, 6, 33-52. doi:10.1080/107490399095247 12

Nystrand, M., Wu, L. L., Gamoran, A., Zeiser, S. \& Long, D. A. (2003). Questions in time: Investigating the structure and dynamics of unfolding classroom discourse. Discourse Processes, 35, 135-198. doi:10.1207/S15326950DP3502_3

Preiss, D. D. (2009). The Chilean instructional pattern for the teaching of language: A video-survey study based on a national program for the assessment of teaching. Learning and Individual Differences, 19, 1-11. doi:10.1016/j.lindif.2008.08.004

Preiss, D. D. (2010). Folk pedagogy and cultural markers in teaching: Three illustrations from Chile. En D. D. Preiss \& R. J. Sternberg (Eds.), Innovations in educational psychology: Perspectives on learning, teaching, and human development (pp. 325-356). New York, NY: Springer.

Sinclair, J. M. H. \& Coulthard, M. (1975). Towards an analysis of discourse: The English used by teachers and pupils. London, Reino Unido: Oxford University Press.

Stigler, J. W., Gallimore, R. \& Hiebert, J. (2000). Using video surveys to compare classrooms and teaching across cultures: Examples and lessons from the TIMSS video studies. Educational Psychologist, 35, 87-100. doi:10.1207/S15326985EP3502_3

Stigler, J. W., Gonzales, P., Kawanaka, T., Knoll, S. \& Serrano, A. (1999). The TIMSS videotape classroom study: Methods and findings from an exploratory research project on eighth-grade mathematics instruction in Germany, Japan, and the United States. Washington, DC: U.S. Department of Education, National Center for Education Statistics.

Stigler, J. W. \& Hiebert, J. (1999). The teaching gap: Best ideas from the world's teachers for improving education in the classroom. New York, NY: The Free Press. 
Tinsley, H. E. A. \& Brown, S. D. (2000). Handbook of applied multivariate statistics and mathematical modelling. San Diego, CA: Academic Press.

Valenzuela, J. P., Bellei, C., Sevilla, A. \& Osses, A. (2009). ¿Qué explica las diferencias de resultados PISA Matemática entre Chile y algunos países de la OCDE y América Latina? En L. Cariola, G. Cares \& E. Lagos (Eds.), ¿Qué nos dice PISA sobre la educación de los jóvenes en Chile? (pp. 105-148). Santiago, Chile: Ministerio de Educación, Unidad de Currículum y Evaluación.

Fecha de recepción: Noviembre de 2009.

Fecha de aceptación: Octubre de 2010.
Wells, G. (1996). Using the tool-kit of discourse in the activity of learning and teaching. Mind, Culture, and Activity, 3, 74-101. doi:10.1207/ s15327884mca0302_2

Wells, G. (1999). Dialogic inquiry: Towards a sociocultural practice and theory of education. New York, NY: Cambridge University Press.

Wells, G. \& Mejia Arauz, R. (2006). Dialogue in the classroom. The Journal of the Learning Sciences, 15, 379-428. doi:10.1207/s15327809jls1503_3 